\title{
From EXILL (EXogam at the ILL) to FIPPS (FIssion Product Prompt $\gamma$-ray Spectrometer)
}

\author{
A. Blanc ${ }^{1, a}$, A. Chebboubi ${ }^{1,2}$, G. de France ${ }^{3}$, F. Drouet ${ }^{2}$, H. Faust ${ }^{1}$, M. Jentschel ${ }^{1}$, G. Kessedjian ${ }^{2}$, U. Köster $^{1}$, S. $^{2}$ \\ Leoni $^{4}$, T. Materna ${ }^{5}$, P. Mutti ${ }^{1}$, S. Panebianco ${ }^{5}$, C. Sage ${ }^{2}$, G. Simpson ${ }^{2}$, T. Soldner ${ }^{1}$, C. A. Ur ${ }^{6}$, W. Urban ${ }^{1,7}$, and A. \\ Vancraeyenest $^{2}$ \\ ${ }^{1}$ Institut Laue-Langevin, France \\ ${ }^{2}$ Laboratoire de Physique Subatomique et de Cosmologie, CNRS/IN2P3, UJF, INPG, France \\ ${ }^{3}$ Grand Accélérateur National d'lons Lourds (GANIL), CEA/DSM - CNRS/IN2P3, B. P. 55027, F-14076 Caen Cedex 5, France \\ ${ }^{4}$ INFN Sezione di Milano, I-20133 Milano, Italy \\ ${ }^{5}$ DSM/IRFU/SPhN, CEA Saclay, France \\ ${ }^{6}$ INFN Legnaro, 35020, Legnaro (Pd), Italy \\ ${ }^{7}$ Faculty of Physics, University of Warsaw, ul. Pasteura 5, 02093 Warsaw, Poland
}

\begin{abstract}
Within the EXILL campaign a large and efficient cluster of Ge-detectors was installed around a very well collimated neutron beam. This has allowed to carry out rather complete spectroscopic studies close to the line of stability using the (n, $\gamma$ ) reaction. Neutron rich isotopes were produced by neutron induced fission and prompt spectroscopy was carried out. The isotope selection in this setup was based on a partially known level scheme and the use of triple coincidences. The latter is limiting the statistical sensitivity in the case of weak production yields. Based on the experiences of these campaigns we are currently developing a new setup: FIPPS (FIssion Product Prompt Spectroscopy). This setup combines a collimated neutron beam, a highly efficient cluster of Ge detectors, a gas filled magnet and auxiliary detectors. The presence of the gas filled magnet will allow us to identify fission products directly and should give access to a new quality of studies if compared to the EXILL campaign. The EXILL campaign and the FIPPS project are presented.
\end{abstract}

\section{Introduction}

Accurate thermal neutron-induced fission data is important for both fundamental nuclear physics and application in reactor physics. Indeed both, the design of Generation IV reactors or reactors based on the thorium cycle require information on transmutation of nuclear waste to fission products. The modeling of the fission process itself requires fission observables from various fissile systems to be measured. In addition, the study of neutron-rich nuclei produced in such reactions is of importance for nuclear structure which, nowadays, aims at the understanding how nuclear potential parameters change far from stability. The study of neutron-rich nuclei is also important for the understanding of the creation of heavy elements in the universe by means of the astrophysical r-process. Thermal neutron induced fission is an important tool for studying nuclear fission and for producing a large variety of neutron rich isotopes far from stability.

In 2012, the EXOGAM high efficiency Ge array [1] has been installed for two ILL reactor cycles (100 days) at the intense cold neutron beam line PF1B at the ILL in order to perform a set of experiments devoted to nuclear spectroscopy: the EXILL (EXogam at the ILL) mea-

\footnotetext{
ae-mail: blanc@ill.fr
}

surement campaign. The spectroscopy of very neutronrich isotopes from neutron induced fission of ${ }^{235} U$ and ${ }^{241} P u$ was performed via $\gamma-\gamma$ or $\gamma-\gamma-\gamma$ coincidences. Mass and nuclear charge were identified via coincidences with known $\gamma$-rays of the nucleus of interest or its complementary fragment (mass cannot be uniquely resolved due to neutron evaporation).

However, where this is not possible, an additional mass information from an ancillary spectrometer can improve the overall resolving power to identify new, weak $\gamma$-rays. In addition, advanced fission studies require the determination of mass and kinetic energy of the fission fragments combined with a simultaneous detection of prompt $\gamma$-rays and possibly also fission neutrons.

In this context, FIPPS a new FIssion Product Prompt $\gamma$ ray Spectrometer is being developed at the ILL. The FIPPS project actually derives from the EXILL campaign setup, in a sense that its goal is to combine a high-resolution $\gamma$ ray spectroscopy together with a large acceptance recoil spectrometer. In a first part the EXILL campaign will be presented. In a second part, the FIPPS project will be introduced. 


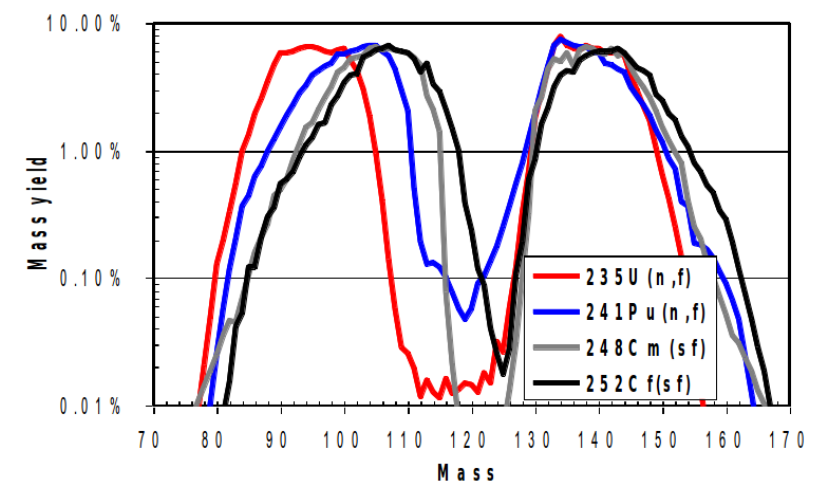

Figure 1. Fission fragments mass distribution following the neutron induced fission of ${ }^{235} \mathrm{U}$ (in red) and ${ }^{241} \mathrm{Pu}$ (in blue), and the spontaneous fission of ${ }^{248} \mathrm{Cm}$ (in grey) and ${ }^{252} \mathrm{Cf}$ (in black).

\section{From EXILL (EXogam at the ILL) ...}

Nowadays, performing $\gamma$-ray spectroscopy of fission products following the spontaneous fission is the most efficient method for studying low-lying excited states of neutronrich nuclei. It allows to study the nuclear structure of about 250 nuclei in the mass range $A=85-160$. Efficient arrays of Ge detectors, such as EUROGAM/EUROBALL [2] or GAMMASPHERE, allow $\gamma$-ray coincidences analysis from which decay schemes are extracted by means of $\gamma-\gamma$ or $\gamma-\gamma-\gamma$ coincidences. However, only the ${ }^{252} C f$ and ${ }^{248} \mathrm{Cm}$ spontaneous fission sources are available for such experiments.

In order to extend the number of accessible nuclei, one can produce very neutron-rich nuclei using the neutron induced fission process. Indeed the fission reaction can be induced in many actinide targets using thermal or cold neutrons. Since the prompt-neutron evaporation following the neutron induced fission is very low, the fission fragments remain very neutron-rich. About ten different fissile targets are available to perform such experiments and amongst them, the ${ }^{235} \mathrm{U}$ and ${ }^{241} \mathrm{Pu}$ targets are the most interesting. The figure 1 displays the fission fragments mass distribution following the spontaneous fission of ${ }^{248} \mathrm{Cm}$ and ${ }^{252} C f$ (in grey and black respectively) and the neutron induced fission of ${ }^{235} \mathrm{U}$ (in red) and ${ }^{241} \mathrm{Pu}$ (in blue). It shows that ${ }^{235} \mathrm{U}$ and ${ }^{241} \mathrm{Pu}$ targets give access to a large amount of nuclei which have currently not been studied in detail. In particular they allow to study the regions northeast of the ${ }^{78} \mathrm{Ni}$ and beyond ${ }^{132} \mathrm{~S} n$ which are of particular interest due to their doubly-magic structure.

The EXILL campaign aimed at combining the intense cold neutron beams available at the Institut Laue-Langevin (ILL) with the EXOGAM high efficiency Ge detector array in order to perform $\gamma$-ray spectroscopy experiments of $21(\mathrm{n}, \gamma)$ targets $[3,4]$ and of the fission fragments following the neutron induced fission of ${ }^{235} \mathrm{U}$ and ${ }^{241} \mathrm{Pu}$ samples. The EXILL campaign was spread over two ILL reactor cycles, for a total of around 100 days. During 30 days of the second cycle $16 \mathrm{LaBr}_{3}(\mathrm{Ce})$ scintillators from the FATIMA collaboration were combined with the 8 EXOGAM detectors for fast-timing measurements [5]. Here

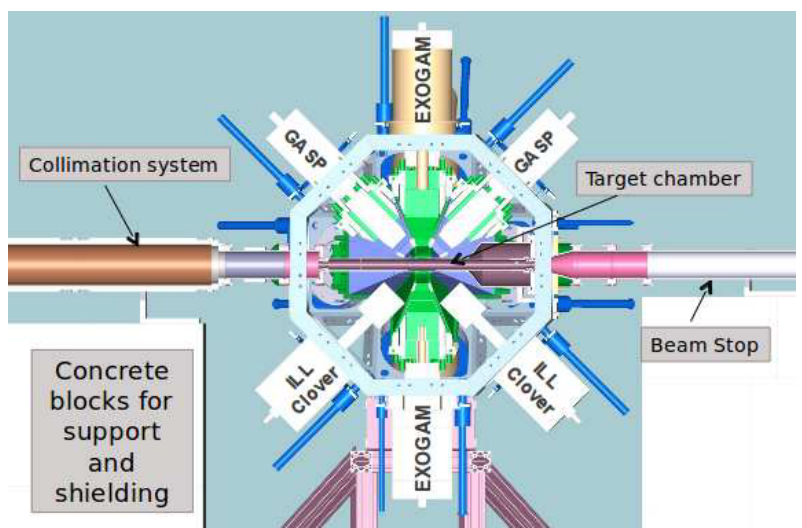

Figure 2. Schematic view of the EXILL setup.

the EXILL setup and some key performances with actinide targets will be presented.

\subsection{The EXILL setup}

The EXILL campaign took place at the ILL PF1B beam line at the end position of the $\mathrm{H} 113$ ballistic neutron guide [6]. The capture flux of the uncollimated beam is $2.10^{10}$ neutrons. $\mathrm{cm}^{-2} \cdot \mathrm{s}^{-1}$. The beam size of $20 \times 6 \mathrm{~cm}^{2}$ at the guide exit has been reduced to match a diameter of about $1 \mathrm{~cm}$ at the sample position by means of a new collimation system [7]. It avoids neutron capture on the beam tube and surrounding material mainly made of aluminium and significantly reduces the $\gamma$ background, allowing closer geometry for the Ge detectors. The measured flux at the target position was $10^{8}$ neutrons. $\mathrm{cm}^{-2} . \mathrm{s}^{-1}$.

The outer target chamber was made of an aluminium pipe of $50 \mathrm{~mm}$ diameter and $2 \mathrm{~mm}$ wall thickness to minimise $\gamma$ absorption. In order to absorb remaining scattered neutrons and reduce neutron induced background, the chamber was surrounded with a $1 \mathrm{~mm}$ thick $6 \mathrm{LiF}$ rubber. For safety reason, an inner chamber made of an aluminium tube of $35 \mathrm{~mm}$ inner diameter and $2 \mathrm{~mm}$ wall thickness was added for the ${ }^{241} \mathrm{Pu}$ runs. A more precise description of both inner and outer target chambers can be found in [8].

All of the actinide targets were sandwiched between dense backing to ensure rapid stopping of the fission fragments. It allowed an almost Doppler-free measurement of the emitted $\gamma$-rays, improving the $\gamma$ energy resolution achieved with the setup. During the overall EXILL campaign, three actinide targets were placed inside the chamber for fission fragment spectroscopy: one ${ }^{235} U, 99.7 \%$ enriched $(525 \mu \mathrm{g})$ with a $15 \mu \mathrm{m}$ thick $\mathrm{Zr}$ backing, one ${ }^{235} \mathrm{U}$, $99.7 \%$ enriched $(675 \mu \mathrm{g})$ with a $25 \mu \mathrm{m}$ thick Be backing and a ${ }^{241} \mathrm{Pu}, 78.6 \%$ enriched $(300 \mu \mathrm{g})$ with a $25 \mu \mathrm{m}$ thick Be backing.

The EXILL setup consisted of a total of 16 Ge detector units: 8 EXOGAM segmented clover detectors from GANIL, 2 non-segmented clover detectors from the LOHENGRIN mass separator at the ILL and 6 GASP n-type $80 \%$ relative efficiency coaxial detectors from LNL Legnaro [9]. Apart from the ILL detectors, all of them were 
equipped with active BGO (bismuth germanate) Comptonsuppression shields. BGO and Ge signals were recorded together allowing off-line anti-coincidence discrimination. The figure 2 displays a schematic view of the EXILL setup. It shows the placement in space of all the detectors around the target chamber: the 8 EXOGAM clovers were placed at $90^{\circ}$ to the beam in a single ring in the perpendicular plane with respect to the beam axis, the 2 LOHENGRIN clovers were placed parallel to the beam axis, at $45^{\circ}$ below the sample and the 6 GASP were filling all the remaining positions at $45^{\circ}$. The segmentation of the EXOGAM detectors was not used during the EXILL campaign.

The EXILL data were recorded in a fully digital and triggerless mode. The high rates achieved (up to 1 $\mathrm{MHz}$ ) required a new acquisition system to be developed [10]. The detectors analogue signals were sampled by ten V1724, 8-channel, 14-bit, 100-MS/s CAEN digitiser cards [11]. Both amplitude of the analogue signals and their arrival in time were recorded by two RIO3 PowerPC-based VME single board computer [12]. The data acquisition system was handling $68 \mathrm{Ge}$ and BGO channels and up to 72 when the FATIMA detectors were put in place.

\subsection{Some key performances}

During the measurement over two reactor cycles over $95 \%$ of the available beam time was used for measurements, the remainder needed for configuration changes and the fixing of few technical problems. Hence, about 60 TB of triggerless data has been recorded. Between two runs with different targets calibration measurements were performed with radioactive sources (activity calibrated ${ }^{60} \mathrm{Co}$ and ${ }^{152} \mathrm{Eu}$ sources) and (n, $\gamma$ ) measurements with a $\mathrm{BaCl}_{2}$ source for the high energy calibration. The long runs with actinide targets (5 to 15 days) were not interrupted for calibration measurements. However, the capture of scattered neutrons on structural materials provides known $\gamma$-rays during the fission runs. Such $\gamma$-ray lines mainly come from ${ }^{27} A l(\mathrm{n}$, $\gamma)$ and ${ }^{27} A l(\beta \gamma)$ and allows and allow to check the energy calibration and energy resolution over time. More detailed calibration and energy resolution performances are reported in [8]. The EXILL detector array efficiency is discussed in [13].

Data evaluation was performed online in order to check the quality of the data sets. The best way is to construct a coincidence matrix, gate on two $\gamma$-ray from a nucleus whose excitation scheme is known and compare the resulting gated $\gamma$-ray spectrum with existing data. In order to illustrate the very good quality of the EXILL data, especially in terms of statistics, we have chosen to use the ${ }^{92} R b$ nucleus and a EUROGAM data set since the $\gamma$-ray efficiencies of the EUROGAM array was very similar to the EXILL one. The figure 3 (a) displays the $\gamma$-ray spectrum gated on the $142 \mathrm{keV}$ and $734 \mathrm{keV}$ low-lying transitions from a 10 days EUROGAM data set with a ${ }^{248} \mathrm{Cm}$ spontaneous fission source [2]. In the same way, the figure 3 (b) displays the $\gamma$-ray spectrum gated on the same transitions obtained from a 4 hours data set of the ${ }^{235} U(\mathrm{n}, \mathrm{f}) \mathrm{EX}-$ ILL measurement (less than $1 \%$ of the total ${ }^{235} U(\mathrm{n}, \mathrm{f})$ data set). Despite the factor 60 in acquisition time in favour of the EXILL data, they show very similar results in terms of statistics which illustrates the very good quality of the data recorded during the EXILL campaign.

\section{3 ... to FIPPS (FIssion Product Prompt $\gamma$-ray Spectrometer}

\subsection{The FIPPS layout}

The EXILL campaign potential physics output, from both $(\mathrm{n}, \gamma)$ and fission targets, is substantial, and the interest of the nuclear structure community in such kind of experiments is clear. The very large number of scientific proposals (more than 80) submitted in the framework of the EXILL campaign very well illustrates this statement. A few proposals were submitted to study the fission process itself [14] but the lack of any way to clearly identify the fission events makes such data analysis quite challenging. Actually, the determination of the mass and kinetic energy of the fission fragments is fundamental to study the fission process.

In addition, as mentioned in section 2, the spectroscopy of very neutron-rich isotopes using fission products is performed via complex $\gamma-\gamma-\gamma$ coincidences analysis which requires for a nucleus to be studied that at least two transitions are known. An additional information in the mass of one of the two fragments would improve significantly the capability of a Ge detector array to identify new $\gamma$-rays.

All these arguments as well as the strong interest of both fission and nuclear structure communities are in favor of the development of a new instrument at the ILL combining an EXILL-like Ge detector array with a recoil spectrometer. As it was during the EXILL campaign, the FIPPS instrument will be used with both $(n, \gamma)$ and fission targets. However, the fission target will not be sandwiched between dense backing but only be backed on a single side. In this way, the first fragment will be stopped in the target backing allowing a Doppler-free detection of the prompt $\gamma$-rays and the second fragment will fly to the spectrometer for mass and kinetic energy measurements.

The FIPPS project is one of 7 new instruments to be built in the frame of ILL's modernisation program called "ENDURANCE". The installation of FIPPS will proceed in three phases, as outlined in the following:

\subsection{FIPPS phase I}

During the first phase, only the Ge detector array will be installed. Measurements will start using (n, $\gamma)$ targets which are, from the safety point of view, easier to manipulate than actinides. After one or two cycles of operation with such targets, measurements using ${ }^{233} U$ or ${ }^{235} U$ targets will start.

The setup will be very similar to the EXILL campaign one. A single ring of clover detectors will be used as a basis and remaining space around the target will be filled with additional detectors depending on the 

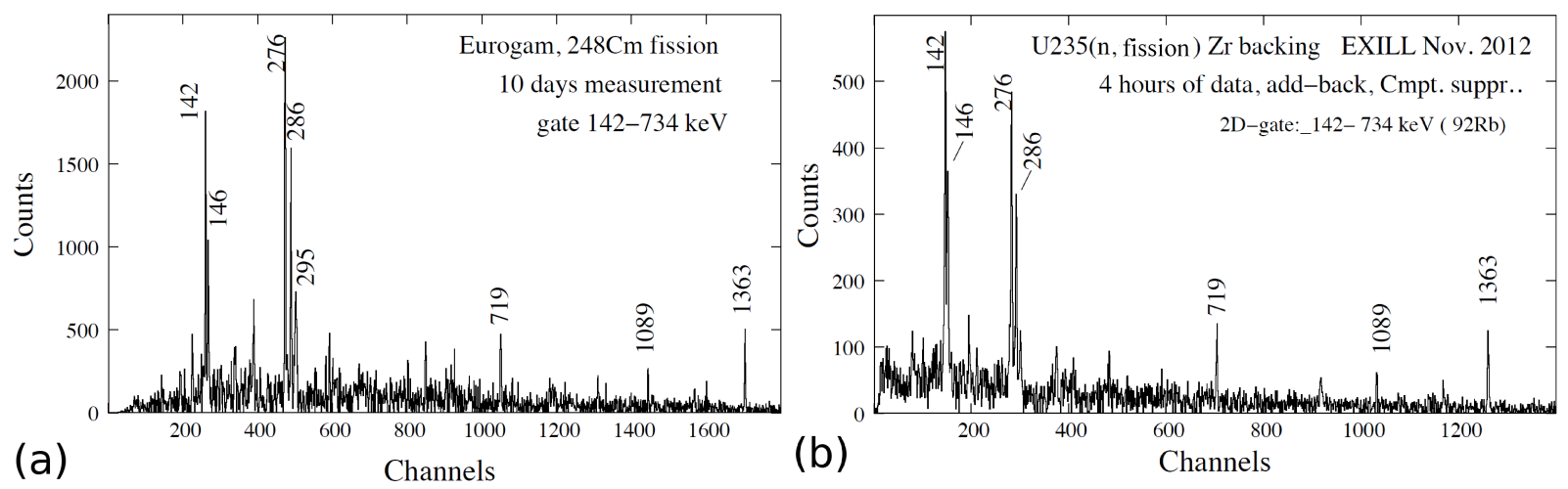

Figure 3. $\gamma$-ray spectrum gated on the ${ }^{92} R b$ low-lying transitions at $142 \mathrm{keV}$ and $734 \mathrm{keV}$ from the ${ }^{248} \mathrm{Cm}$ EUROGAM data (a) [2] and ${ }^{235} U(\mathrm{n}, \mathrm{f})$ EXILL data (b).

current experiment's needs. For instance the array efficiency could be improve by adding Ge detectors such as the LOHENGRIN's clovers in order to study low cross section processes. Installing BEGe (Broad Energy Germanium) detectors could significantly improve the energy resolution at very low energy, allowing to study very weak low energy transitions. Such detectors would also provide an additional X-ray identification. As in the EXILLFATIMA campaign the setup can also be complemented by $\mathrm{LaBr}_{3}(\mathrm{Ce})$ detectors for ultrafast timing studies. Several other options are also considered such as plunger or g-factor measurements, or even prompt neutron detection which are of importance for the study of the fission process.

For efficient fission studies, a fission trigger is essential to distinguish between fission $\gamma$-rays and capture $\gamma$-rays. In addition such a device would allow to efficiently clean the $\gamma$ spectra from $\beta$-decay induced background. Used as a veto, it would also allow to measure (n, $\gamma$ ) cross sections on fissile material. Usual fission triggers/veto use fission targets in a counting gas or in a vacuum chamber equipped with fission detectors. Such combinations are in principle possible but render the installation inside the Ge detector array more complex. To deal with it, a new kind of active target is under development at CEA DAM/DIF for which the fissile material is mixed with an organic liquid scintillator. Such a technique combines the advantage of reaching very high actinide concentrations (up to $10 \mathrm{mg} / \mathrm{mL}$ of ${ }^{235} \mathrm{U}$ ) with the intrinsic organic scintillator capability: the fast time response and the Pulse Shape Discrimination (PSD) for $\alpha-\beta$-fission product separation. Moreover such active targets are easy to make, most of the organic liquid scintillators being available commercially. The performances of two prototypes are reported in [15]. However, the ageing behavior when exposed to a high fission product rate has never been studied so far and an experiment is ongoing using a high flux test instrument of the ILL.

The FIPPS project requires a thermal or cold neutron beam with a $\sim 10^{8} \mathrm{n} \cdot \mathrm{cm}^{-2} \cdot \mathrm{s}^{-1}$ flux and a cross-section of approximately $10 \times 10 \mathrm{~mm}^{2}$. The instrument has to be sited at the end position of a neutron beam since the beam quality downstream of the target and spectrometer does not allow further use for neutron scattering applications. In addition, the Ge detector array is very sensitive to $\gamma$ ray background and moderately sensitive to neutron background. A suitable position is already available at ILL.

Thanks to the EXILL campaign no additional technical development is needed and, in this sense, the EXILL campaign has also been an important step for the development of FIPPS. Indeed both, a triggerless DAQ capable of handling $1 \mathrm{MHz}$ event rate and the collimation system allowing the safe operation of Ge detectors close to an intense neutron beam are already in place at ILL.

\subsection{FIPPS phase II}

In the second phase, the spectrometer will be built to extend the capability of the Ge detector array. Dedicated recoil separators such as LOHENGRIN at ILL (doublefocusing parabola spectrometer) [16] provide a physical fragment separation with excellent mass and energy resolution. They allow precise $\gamma$ decay spectroscopy of $\mu s$ isomers and $\beta$-decaying isotopes. But such separators suffer from a very small acceptance $(<0.01 \%)$ which makes them incompatible with prompt $\gamma$-ray spectroscopy at the target position.

FIPPS is designed to allow precise prompt $\gamma$-ray spectroscopy, making FIPPS and LOHENGRIN complementary. It is based on the GasFilled Magnet (GFM) separation method. This method has been developed in Oak Ridge in 1958 [17] and has been used in the last decades for the construction of recoil separators. Some example of GFM are JOSEF (Jülich Online SEparator for Fission products) built at the reactor FRJ-2 in Jülich in 1973 [18] or the recent TASCA (TransActinide Separator and Chemistry Apparatus) for super-heavy element studies at GSI [19]. In a magnetic dipole under vacuum, fission fragments are separated according to the ratio $\frac{p}{Q}$ where $p$ is the fragment's momentum and $Q$ its ionic charge. When the dipole is filled with a gas, the fission fragments interact with the gas through charge exchange, energy loss or scattering, and this formula is no longer valid. However, 
if the mean free path between two charge exchange collisions is small enough, it has been shown in [17] that the magnetic rigidity $B \rho$ is proportional to $\frac{A}{Z^{\alpha}}$ with $\mathrm{A}$ and $\mathrm{Z}$ the fragment's nuclear mass and charge respectively. The parameter $\alpha$ depends on the gas and pressure. This leads to the main feature of the GFM spectrometers: the fission fragments trajectory no longer depends on the initial ionic charge nor on the velocity (to the first order). This feature allows to maximise the transmission of the spectrometer.

The development of the FIPPS spectrometer is ongoing. Preparatory experiments at LOHENGRIN and one conceptual designares presented in detail in [20]. The unique concept of this design is to use the intrinsic ionization in the gas to perform individual 3D-tracking of the fragments. In this way, all the fragments can be detected simultaneously inside the spectrometer allowing EXILLlike measurements where all fragments are measured at the same time.

\subsection{FIPPS phase III}

The third phase of the FIPPS project consists in a relocation to a newly built casemate that will allow a safe routine use of transuranium targets $\left({ }^{239} \mathrm{Pu},{ }^{241} \mathrm{Pu},{ }^{242} \mathrm{mAm}\right.$, ${ }^{243} \mathrm{Cm},{ }^{245} \mathrm{Cmm},{ }^{247} \mathrm{Cm},{ }^{249} \mathrm{Cf},{ }^{251} \mathrm{Cf}$ ). Such casemate will considerably extend the number of nuclei accessible for nuclear structure studies.

\subsection{Conclusion}

In 2012-2013, during two reactor cycles ( $\sim 100$ days), the EXOGAM Ge detector array was moved at the ILL PF1B beam line for a series of nuclear physics experiment: the EXILL campaign. In total, 24 different samples have been irradiated, including ${ }^{235} \mathrm{U}$ and ${ }^{241} \mathrm{Pu}$ samples. A dedicated collimation system allowing the safe operation of a Ge array close to an intense neutron beam has been developed. Data was recorded using a fully digital, triggerless, acquisition system capable of handling an event rate of about $1 \mathrm{MHz}$.

The EXILL campaign has been an important step for the development of the new ILL project FIPPS. FIPPS is part of the ILL ENDURANCE program aiming at upgrading the ILL's instrument suite. The ENDURANCE program has been officially launched in 2014 and the first Ge detectors are planned to be ordered soon.

\section{Acknowledgement}

The EXILL campaign would not have been possible without the support of several services at the ILL and the LPSC. We are grateful to the EXOGAM collaboration for the loan of the detectors, to GANIL for assistance during installation and dismantling, and to the INFN Legnaro laboratory for the loan of the GASP detectors.

\section{References}

[1] J. Simpson et al., Acta Phys. Hung., New Ser. Heavy Ion Phys. 11, 159 (2000)

[2] W. Urban et al., Phys. Rev. C 85, 014329 (2012)

[3] J. Jolie et al., these proceedings (2014)

[4] P. Baczyk et al, these proceedings (2014)

[5] J.-M. Régis et al., these proceedings (2014)

[6] H. Abele et al., Nucl. Instr. Meth. A, 562, 407 (2006)

[7] W. Urban et al., JINST, 8, 3014 (2013)

[8] A. Blanc et al., EPJ Web of Conf. 62, 01001 (2013)

[9] C. Rossi Alvarez, Nucl. Phys. News, 3, no. 3 (1993)

[10] P. Mutti et al., Proc. ICALEPCS2011, 935 (2011)

[11] CAEN (2010), Italy, Technical information manual mod. V1724 8 channel 14 bit $100 \mathrm{MS} / \mathrm{s}$ digitiser, [Online], Available : http ://www.caen.it

[12] CES (2003), Switzerland, RIO3 8064 powerpc-based VMEx-LI processor board user manual DOC 8064/UM version 3.4, [Online], Available : http ://www.ces.ch

[13] C. Lorenz et al., these proceedings (2014)

[14] T. Materna et al., these proceedings (2014)

[15] G. Belier et al., Nucl. Instr. Meth. A, 664, 341 (2012)

[16] G. Siegert et al., Phys. Lett. 53B, 45 (1974)

[17] B. L. Cohen and C. B. Fulmer, Nucl. Phys. 6, 547 (1958)

[18] P. Armbruster et al., Nucl. Instr. Meth, 137, 103 (1976)

[19] J. Even et al., Nucl. Instr. Meth. A, 638, 157 (2011)

[20] A. Blanc et al., Nucl. Instr Meth. B, 317, 333 (2013) 
